\title{
Study on Research Development and Application of Urban Logistics Platform Based on Android
}

\author{
Wu Junliang, Mao Liqing \\ Institute of Technology. East China Jiaotong University, Nanchang Jiangxi, 330100, China
}

Keywords: urban logistics platform; Android; development; application

\begin{abstract}
Android is an advanced development language applied to mobile devices, with great prospect for the application to market. This paper analyzes the development and characteristics of Android, and introduces the development and relevant application of urban logistics platform based on Android.

Android is a development language applied to mobile devices. With the popularity of mobile electronic devices, there are more software and embedded devices with Android as development language. With the continuous development of online shop and express delivery business, the importance of establishing urban logistics platform is more urgent, which can build a platform for better information communication and information management between both sides of the logistics. In the era of mobile payment and information inquiry, the development of urban logistics platform based on Android as language basis has a certain significance, which is described specifically in this paper.
\end{abstract}

\section{Android and its Characteristics}

Android, put forward by Google, is an advanced development language applied on mobile devices. Mobiles phones or tablet devices with Android as operating system accounts for one half of all mobile phones or tablet devices. Android is equipped with a whole set of development process and corresponding development tools, with Linux as system kernel and Java as development language. Since then, Android developed greatly, and each new version solved part of problems in old versions and improves the system.

Android is suitable for the development of mobile terminal, and there are many advantages in the development of software with Android as development platform, with flexible, portable and simple characteristics. And with the development of Android, the learning of Android is easier for learners to learn relevant knowledge in community of Android, which reduces the learning cost of Android. On the whole, Android is suitable for the development of mobile terminal software. In addition, Android improves itself in each version update, which indicates that there is great prospect for the application of Android.

\section{The Design of Urban Logistics Platform}

In order to ensure the effectiveness of platform development, before actual development, the industry-university cooperation is conducted with Jiangxi Province Ganzhou Lala Logistics Co., Ltd to design logistics platform and ensure that the development process is consistent with expected direction, and the urban logistics platform is temporarily called "lala 98.cn" (hereinafter referred to as logistics platform). The design of logistics platform is described from several aspects.

\subsection{Overall design}

Before the design of logistics platform, it is divided into several logic parts. In this way, first of all, it can be developed more comprehensively, which can better reduce problems in the development process, called one mechanism to reduce development vulnerabilities. Secondly, it is suitable for the development characteristics of Android. MVC is one development mode of Android 
with the main characteristics of logic division. MVC development mode divides the development of Android to model layer, view layer and control layer, with independence in logic, which is consistent with the development process of MVC and conductive to realize specific development content by virtue of Android development language.

This platform is divided into user, interface, function and data.

\subsection{Demand analysis}

More detailed parts are divided in large logic parts, and relevant personnel shall be required to conduct demand analysis of logistics platform and grasp demand of logistics platform. The content of sub-division of urban logistics platform is described.

User. There are different people using logistics platform, including shipper (administrator and general staff), receiving party, logistics party, third party, supervision and administration party of logistics platform, and maintenance party of logistics platform. And there are different demands for different parties, therefore, the authority of system access is different. When finishing user part, a set of general interface shall be set in the system to accomplish information inquiry and setting of related function.

Interface. The interface has a great impact on the development of logistics platform. Outstanding interface is conductive to attracting core users, which is necessary for the development of logistics platform. With the further development of front end, more interface frameworks are applied to urban logistics platform software, and interface performance attracts the attention from more people. An outstanding interface not only requires beautiful appearance, also requires comfortable user experience. Based on market situation and actual requirements, this logistics platform selects react native as interface design framework with better interactivity, which can provide better user experience.

Function. The function is an important part in logistics platform, and the function of software is the main factor influencing evaluations from users. According to different users, software is divided into "shipper" and "driver", which is shown in five aspects. First of all, the personalization function. Personalized function is provided for different users so that users can set their information and change between "shipper" and "driver" to better use the software. Secondly, the function of communication. Different users can communicate through logistics platform, including instant communication and message communication. Besides, in communication process, users also can accomplish the setting of part information. Thirdly, the tacking of cargo. Cargo tracking is a significant part of logistics platform, which is mainly provided by the third party of cargo transport. The system also set communication interface to help other users to communicate with the party of cargo transport. Fourthly, the platform function. In order to ensure the better operation of logistics platform, some users and behaviors shall be regulated in the platform. For instance, if some users publish some unreal or bad information, other users can report to the platform. Then the platform is required to inspect reported content based on reported information, and deal with it based on the results of inspection. In addition, the operation of platform shall satisfy some guidelines of the country. When the country publish new operation standard, it is necessary for the platform to response to standards and make improvements. Fifthly, the inquiry and other functions. Users need inquire information based on use demands. In addition to traditional inquiry of order and logistics information, the software also provide the inquiry of nearby shops, which is convenient for the life of local residents and increases the viscidity of users for the software. Besides, the platform provides rating function. After finishing logistics transactions, the entrusting party can evaluate the transaction, which can not only provide reference for other entrusting parties, also an effective supervision on shippers, so as to ensure the continuous and credible use by users.

Data. The normal operation of logistics platform include many data. In the design of logistics platform, better performance of data is ensured, including reasonability and relatively low redundancy. Besides, data storage and means of transportation shall be well designed to guarantee that logistics platform can provide instant, stable and secure information. In order to ensure data security, the initial design of the software include three parts. First of all, physical redundancy. 
Information distortion may occurs in transportation and storage of information. In order to solve this problem, the method of physical redundancy is designed to store many copies of core data, and conduct multi-votes in analysis and storage of data to ensure the uniformity of information. Secondly, distributed data storage. Distributed data storage can better guarantee data security, because when the content of one database is corrupted, other databases can provide correct information. Besides, distributed data storage can provide better experience for users. When one information channel is overloaded with information because of information explosion or other reasons, other channels can provide information transmission. Thirdly, information security. Information security is a necessary part to be focused in network design. In the design of logistics platform, encryption algorithm, security verification and anti-attack system provide certain security guarantee. At present, the first and third requirements have been achieved preliminarily, and in further development, if there is demand in volume of business, distributed data storage will be conducted.

\subsection{Design objectives}

Design objectives of the software include three aspects.

First of all, high reliability. High reliability guarantees the reliability of user information and security of user data.

Secondly, usability. In the design of logistics platform, certain staff shall be arranged to conduct the analysis and survey on the market to ensure that function design and interface logic design of logistics platform can satisfy usage requirements and using habit of users.

Thirdly, expandability. The expandability of logistics platform is a key part of the platform design, because with the development of logistics platform and the society, logistics platform is required to add, eliminate and improve some functions, to be more consistent with times changes and satisfy requirements of staff. Therefore, in the design of logistics platform, relevant personnel is required to divide it logically to reduce logic coupling, and to standardize the platform design to guarantee logistics platform code easy to understand, modify and maintain.

\section{The Development of Logistics Platform}

The development of logistics platform with Android language has certain development cycle, which is introduced in this paper.

\subsection{Preparation phase}

In preparation phase of the development, the designer and actual developer should cooperate together. First of all, run-in of both sides. It is necessary for both sides to understand each other. We have discussed specific matters with Jiangxi Province Ganzhou Lala Logistics Co., Ltd and have obtained consensus. Secondly, clear design method of Android. The design method of Android of the logistics platform is Android Studio design software, react native front-end design framework and Cache and DMA used together to improve access performance of data and the application of MySQL lightweight relational database.

\subsection{Design phase}

The design of logistics platform with Android is divided into two parts, the client and server, which are separated logically. Information of client is displayed according to the authority and setting of users, whereas, information of server is a logic processing of information of client, and server needs to give feedback to requirements of client and manage database.

The functions completed in the design of logistics platform with Android include three aspects.

First of all, login, register and logout of users. When users provide certain information, they can register in different way. After the registration, they can login and logout. However, after login, users can set or reset their own information.

Secondly, the improvement of inquiry function. The improvements of inquiry function include the inquiry function of client and server. For the improvements of inquiry function of client, 
Android is required to provide interface to allow client to search fixed information. In the interface design of client, Android is required to accomplish the logicality of interface. For instance, users can only inquire their own order information, whereas, logistics party can inquire all logistics information they process. For server, the server is required to make judgments on user requests. After the permission to user requests, the server makes use of database process code provided by Android to extract corresponding information and give information feedback to information request in data buffer to avoid re-extracting of users. After the data lifecycle, the server is responsible to completely corrupt data to ensure data security. In inquiry function, the server needs be set with certain self-protection measures. For example, when malicious host has a large amount of requests, the server may crash, so it is necessary to judge information source with certain algorithm. Therefore, in preliminary phase, based on the consideration of costs, server and bandwidth are rented from Aliyun for use.

Thirdly, instant tracking. Through the positioning analysis with data code, the system can conduct instant tracking on logistics information according to requirements of users, which can be given back to relevant users.

\section{The Application and Maintenance of Logistics Platform}

There probably are some problems in the application of logistics platform, so two aspects shall be focused to reduce and solve these problems as many as possible.

\subsection{The software trial}

In the software trial, logistics platform conducts related survey on users and analysis and improvements on problems proposed to ensure the interactivity and application characteristics of software.

\subsection{The software maintenance}

The soft maintenance include periodic maintenance and maintenance on emergency problems. It is necessary to make analysis on software performance regularly to guarantee operation security of software. However, when emergency problems occur, it is necessary to find out the root of the problem timely and deal with problems rapidly, which can better deal with the influence of problems on software to cause less loss, therefore, necessary plans to prevent problems are prepared before the usage.

\section{Conclusion}

The design of logistics platform with Android as design language includes the design of logistics platform content and application design of Android. The design of platform content include overall design, demand analysis and design objectives. And the application design of Android include preparation phase and design phase. In the software design, it is necessary to cooperate with relevant enterprises, pay attention to industry-university cooperation, with mutual benefit, responsible for the software trial, post maintenance and the development of IOS version in the future.

\section{Acknowledgement}

Fund program: This paper comes from project of Science and Technology Department of Jiangxi Province, Study on Research Development and Application of Urban Logistics Platform Based on Android. Project No. GJJ161556.

\section{References}

[1] Wu Xiaoxia, Meng Kuntai, Zhao Jie et al. The Development of Intelligent Medical Information Interactive Platform Based on Android [J]. Global Market Information Guide, 2017(18):133-133. 
[2] Ding Xufeng. Development and Application of Device Point Inspection System Based on Android Platform [C]// Collected Papers of Metallurgy Association of China Metrology Association in 2017. 2017.

[3] Wang Peng, Xie Lili, Zhou Huilin et al. Design and Development of Logistics Platform Shipper System Based on Android [J]. China CIO News, 2018(2).

[4] Pang Zeping, Fang Chaoxi, Li Guosheng. Design of Logistics Storage Forklift Dispatching System Based on Android [J]. Electronic Technology \& Software Engineering, 2017(6):66-68.

[5] Han Xiaoyan. Research on Development Methods and Application of Mobile APP Based on Android Platform [J]. Computer Knowledge and Technology, 2017, 13(18):71-72. 